Draft Version OCtober 11, 2018

Typeset using LATEX twocolumn style in AASTeX61

\title{
NO LOW-FREQUENCY EMISSION FROM EXTREMELY BRIGHT FAST RADIO BURSTS
}

\author{
M. Sokolowski, ${ }^{1,2}$ N. D. R. Bhat, ${ }^{1,2}$ J.-P. Macquart, ${ }^{1,2}$ R. M. Shannon, ${ }^{1,3,4}$ K. W. Bannister, ${ }^{3}$ R. D.Ekers, ${ }^{3,1}$ \\ D. R. Scott,${ }^{1}$ A. P. Beardsley ${ }^{5}$ B. Crosse, ${ }^{1}$ D. Emrich,${ }^{1}$ T. M. O. Franzen,${ }^{1}$ B. M. Gaensler,${ }^{6,7,8}$ L. Horsley, ${ }^{1}$ \\ M. Johnston-Hollitt,${ }^{1}$ D. L. Kaplan,${ }^{9}$ D. Kenney, ${ }^{1}$ M. F. Morales,${ }^{10,7}$ D. Pallot,${ }^{11}$ G. Sleap,${ }^{1}$ K. Steele, ${ }^{1}$ \\ S. J. Tingay, ${ }^{1}$ C. M. Trott,${ }^{1,7}$ M. Walker, ${ }^{1}$ R. B. Wayth,${ }^{1,7}$ A. Williams, ${ }^{1}$ and C. Wu ${ }^{11}$
}

\footnotetext{
${ }^{1}$ International Centre for Radio Astronomy Research, Curtin University, Bentley, WA 6102, Australia

${ }^{2}$ ARC Centre of Excellence for All-sky Astrophysics (CAASTRO), Australia

${ }^{3}$ Australia Telescope National Facility, CSIRO Astronomy and Space Science, PO Box 76, Epping, NSW 1710, Australia

${ }^{4}$ Centre for Astrophysics and Supercomputing, Swinburne University of Technology, PO Box 218, Hawthorn, VIC 3122, Australia

${ }^{5}$ School of Earth and Space Exploration, Arizona State University, Tempe, AZ 85287, USA

${ }^{6}$ Sydney Institute for Astronomy, School of Physics, The University of Sydney, NSW 2006, Australia

${ }^{7}$ ARC Centre of Excellence for All Sky Astrophysics in 3 Dimensions (ASTRO 3D), Australia

${ }^{8}$ Dunlap Institute for Astronomy and Astrophysics, University of Toronto, ON, M5S 3H4, Canada

${ }^{9}$ Department of Physics, University of Wisconsin-Milwaukee, Milwaukee, WI 53201, USA

${ }^{10}$ Department of Physics, University of Washington, Seattle, WA 98195, USA

${ }^{11}$ International Centre for Radio Astronomy Research, University of Western Australia, Crawley 6009, Australia
}

\section{ABSTRACT}

We present the results of a coordinated campaign conducted with the Murchison Widefield Array (MWA) to shadow Fast Radio Bursts (FRBs) detected by the Australian Square Kilometre Array Pathfinder (ASKAP) at 1.4 GHz, which resulted in simultaneous MWA observations of seven ASKAP FRBs. We de-dispersed the $24 \times 1.28 \mathrm{MHz} \mathrm{MWA}$ images across the $170-200 \mathrm{MHz}$ band taken at 0.5 second time resolution at the known dispersion measures (DMs) and arrival times of the bursts and searched both within the ASKAP error regions (typically $\sim 10^{\prime} \times 10^{\prime}$ ), and beyond $\left(4^{\circ} \times 4^{\circ}\right)$. We identified no candidates exceeding a $5 \sigma$ threshold at these DMs in the dynamic spectra. These limits are inconsistent with the mean fluence scaling of $\alpha=-1.8 \pm 0.3\left(\mathcal{F}_{\nu} \propto \nu^{\alpha}\right.$, where $\nu$ is the observing frequency) that is reported for ASKAP events, most notably for the three high fluence $\left(\mathcal{F}_{1.4 \mathrm{GHz}} \gtrsim 100 \mathrm{Jy} \mathrm{ms}\right)$ FRBs 171020 , 180110 and 180324. Our limits show that pulse broadening alone cannot explain our non-detections, and that there must be a spectral turnover at frequencies above $200 \mathrm{MHz}$. We discuss and constrain parameters of three remaining plausible spectral break mechanisms: free-free absorption, intrinsic spectral turn-over of the radiative processes, and magnification of signals at ASKAP frequencies by caustics or scintillation. If free-free absorption were the cause of the spectral turnover, we constrain the thickness of the absorbing medium in terms of the electron temperature, $T$, to $<0.03\left(T / 10^{4} K\right)^{-1.35}$ pc for FRB 171020.

Keywords: surveys — radiation mechanisms: non-thermal — methods: data analysis — instrumentation: interferometers 


\section{INTRODUCTION}

The origin of the bright, millisecond-timescale emission associated with Fast Radio Bursts (FRBs) remains an open question. Many of the fundamental observational characteristics of these bursts remain stubbornly elusive, a fact exemplified by poor constraints on even the spectral extent of the radio emission. Until very recent FRB detections by Canadian Hydrogen Intensity Mapping Experiment (CHIME/FRB; The CHIME/FRB Collaboration et al. 2018) down to $400 \mathrm{MHz}$ (Boyle et al. 2018), the lowest frequency FRB was observed at $700 \mathrm{MHz}$ (Masui et al. 2015). Despite major efforts (including Coenen et al. (2014), Karastergiou et al. (2015), Tingay et al. (2015), Rowlinson et al. (2016), Keane et al. (2016), Amiri et al. (2017), Chawla et al. (2017) and Burke-Spolaor et al. (2016) to name a few) to date, no FRB emission has been reported below $400 \mathrm{MHz}$ or above $8 \mathrm{GHz}$ (Gajjar et al. 2018). Currently the only published limit on the spectral index below $700 \mathrm{MHz}$ resulting from simultaneous broadband observations is $\alpha<-3\left(\mathcal{F}_{\nu} \propto \nu^{\alpha}\right.$, where $\mathcal{F}_{\nu}$ is fluence at the observing frequency $\nu$ ), for the Parkes FRB 150418 (Keane et al. 2016). Moreover, there have been no coincident detections of FRBs in any other waveband, despite extensive multi-wavelength simultaneous and follow-up observations with optical, IR, X-ray and gamma-ray facilities (Petroff et al. 2015; Scholz et al. 2016; Law et al. 2017; Keane et al. 2016).

The dearth of FRB detections at low radio frequencies $(\leq 400 \mathrm{MHz})$ presents a critical impediment to the analysis of the burst energetics. Burst energies up to $E \sim 10^{35} \mathrm{~J}$ are inferred by integrating the emission across the observing band and assuming the emission is isotropic (Lorimer et al. 2007; Thornton et al. 2013; Bannister et al. 2017). However, reliable spectral measurements based on the 20 FRBs reported by the Commensal Realtime ASKAP Fast Transients (CRAFT) survey (Macquart et al. 2010) on the Australian Square Kilometre Array Pathfinder (ASKAP) at $1.4 \mathrm{GHz}$, show that the average FRB fluence spectrum is steep, with a spectral index $\alpha=-1.8 \pm 0.3$ (Shannon et al. 2018). This indicates that the low-frequency cutoff likely dominates the energetics of the radio emission, notwithstanding the fact that the bursts detected by ASKAP often exhibit patchy spectral structure (Shannon et al. 2018), as does the repeating FRB 121102 (Spitler et al. 2016). High fluences and steep spectral indices of CRAFT FRBs make them ideal targets for low-frequency observations with the MWA.

Low-frequency measurements also provide diagnostics of the plasma along the line of sight to the FRB. In particular, a number of propagation effects potentially influence the spectral characteristics at low frequencies, and their identification in FRB data would place constraints on the burst environment and the properties of the plasma encountered along the ray path. The most obvious two effects are scattering due to inhomogeneities in the plasma (most likely distant from the source, i.e. $\gg 1 \mathrm{pc}$ ), which causes temporal smearing of the signal proportional to $\sim \nu^{-4}$ (Bhat et al. 2004), and free-free absorption by dense, circumburst plasma, whose optical depth scales as $\tau_{\mathrm{ff}} \sim \nu^{-2.1}$.

The characteristics of the low-frequency emission bear heavily on FRB detection rates with future survey facilities such as the low-frequency component of the Square Kilometre Array (SKA_Low; Fender et al. 2015a). The wide fields-of-view (FoVs) accessible by this telescope render it capable of detecting transient phenomena at extremely high rates (Fender et al. 2015b). Moreover, the recent CHIME/FRB detections show that FRBs can be observed at least down to $400 \mathrm{MHz}$ triggering even more interest in lower frequencies and spectral extent of FRB emission.

In this Letter we present the results of an observing campaign undertaken with the Murchison Widefield Array (MWA; Tingay et al. 2013) to detect low-frequency radio emission coincident with the bright FRBs detected by the CRAFT survey at $1.4 \mathrm{GHz}$ (Macquart et al. 2010; Bannister et al. 2017; Shannon et al. 2018). The MWA shadowed (co-tracked) the pointing positions of the ASKAP antennas, so that the precise dispersion measure (DM), time of arrival and the approximate position (typically a $10^{\prime} \times 10^{\prime}$ region) of each burst are all known, greatly reducing the searched volume of parameter space relative to a blind survey.

\section{OBSERVATIONS AND DATA REDUCTION}

\subsection{Observing Strategy}

The observations were made with the MWA whilst the ASKAP antennas observed in the fly's-eye mode ${ }^{1}$ distributed along a certain Galactic latitude (see Bannister et al. 2017). The FoV of the MWA in the frequency band $170-200 \mathrm{MHz}$ is approximately $450 \mathrm{deg}^{2}$ (FWHM 21 ${ }^{\circ}$, enabling nearly full coverage of the ASKAP fly's eye FoV with sensitivity $\gtrsim 50 \%$ of the primary beam. Moreover, the selected frequency band minimizes the effects of pulse broadening (due to scattering) and radio-frequency interference. Figure 1 shows the observing setup during FRB 180324.

\footnotetext{
${ }^{1}$ Individual antennas pointing in different directions and covering $\approx 300 \operatorname{deg}^{2}$ (with 10 ASKAP antennas).
} 


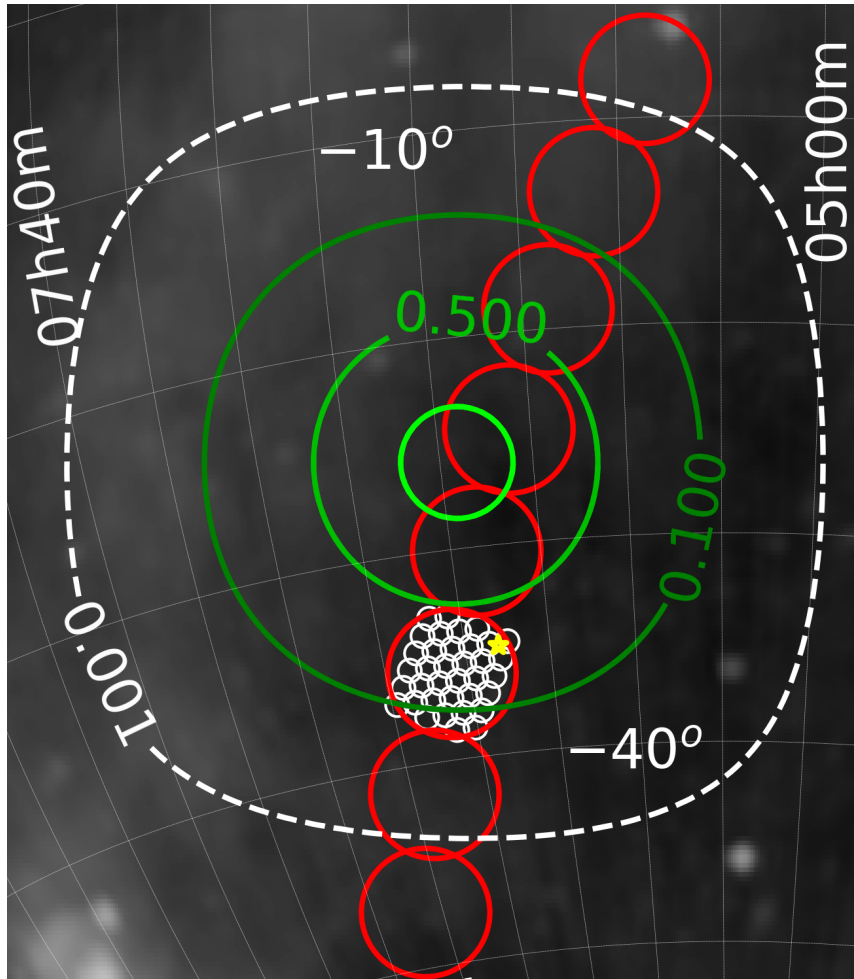

Figure 1. An illustration of the MWA shadowing strategy for FRB 180324 (on top of the image by Haslam et al. (1982) at $408 \mathrm{MHz})$. The large $\left(\approx 7^{\circ}\right)$ red circles represent coverage of ASKAP antenna beams distributed near Galactic latitude $-20^{\circ}$ and small white circles $\left(\approx 1.2^{\circ}\right)$ are the individual 36 beams of ASKAP antenna 25, which detected FRB 180324 (yellow star). The green (at 0.5 and 0.1 ) and white-dashed (at 0.001 with angular size $\approx 42^{\circ} \times 42^{\circ}$ ) contours show the MWA beam (Sokolowski et al. 2017) normalized to maximum response at zenith.

Table 1 summarizes the ASKAP FRBs (Shannon et al. 2018; Macquart et al. 2018, submitted) detected while MWA shadowed. The strategy succeeded for the first time with FRB 171020, and the MWA collected data before, during and after the FRB detection by ASKAP (Table 1). The MWA data were collected during the transition to the extended array (Wayth et al. 2018), implying reduced sensitivity (only $\sim 70 \%$ of antennas). Since then, six more ASKAP FRB positions were observed by the MWA in a similar mode. In all cases MWA data were recorded in $10-\mathrm{kHz}$ frequency and 0.5 -second temporal resolutions.

The shadowing program was performed mostly during daytime. Hence, only FRBs 171020 and 180324 were detected by ASKAP after sunset whilst the FRB 180110 field was observed with the Sun close to a "null" of the MWA's primary beam (a direction with very low sensitivity). In the other cases the data quality was too low to derive meaningful limits due to the presence of the Sun in the sidelobe of the primary beam.

\subsection{Calibration}

The MWA data were calibrated with the Common Astronomy Software Applications (CASA; McMullin et al. 2007), using observations of a calibrator source (3C444 or Pictor A). We applied calibration solutions to FRB field observations and created dirty images in 0.5 -second temporal (shortest possible) and $1.28-\mathrm{MHz}$ frequency resolutions using WSCLEAN (Offringa et al. 2014) and natural weighting.

We also calibrated and imaged MWA observations (292 seconds) collected before the FRB observations in order to create reference images of the FRB fields (typical standard deviation of noise $\sigma \sim$ $20-40 \mathrm{mJy}$ beam $^{-1}$ ). The flux density scale was calibrated using sources from the GaLactic Extragalactic All-sky MWA (GLEAM) survey (Hurley-Walker et al. 2017) identified in the reference images.

\subsection{FRB detection algorithm}

The transient detection algorithm used the reference images (Sec. 2.2) to create a list of reference sources above certain threshold $(5 \sigma)$. The $24 \times 1.28 \mathrm{MHz}, 0.5$ second images across the observing band were timealigned according to the ASKAP DM and summed to provide a 0.5 -second resolution de-dispersed image (example images are shown in Fig. 2). We also examined the de-dispersed images before and after the expected burst arrival time (we inspected all 0.5-s images within the analyzed MWA observation). The details of candidates exceeding a $5 \sigma$ threshold, which were not present in the list of reference sources, were saved for further visual inspection. We did not observe any $>5 \sigma$ event within the ASKAP error boxes ${ }^{2}$.

The $5 \sigma$ transient candidates identified within a larger $\left(4^{\circ} \times 4^{\circ}\right)$ field were visually inspected on de-dispersed images and dynamic spectra of the candidate pixels, and none of them showed any signs of dispersion sweep in the dynamic spectra. As a final check, we visually inspected all 0.5 -second, 1.28- $\mathrm{MHz}$ images.

FRB 180110 was significantly scattered (Tab. 1), with an expected pulse width at the MWA's frequency, $\tau_{\mathrm{MWA}} \approx 5.4$ seconds (Sec. 3). Therefore, in the second part of the algorithm we averaged over multiple dedispersed (and non-dedispersed) images on timescales of $5,10,15,20,25$ and 30 seconds. Yet, we did not identify any transient events exceeding $5 \sigma$ threshold with

\footnotetext{
2 The expected number of candidates exceeding $5 \sigma$ due to Gaussian noise fluctuations is $\ll 1$ for $\sim 100 \times 100$ pixels.
} 
Table 1. The details of the ASKAP FRBs shadowed by the MWA

\begin{tabular}{|c|c|c|c|c|c|c|c|c|c|c|c|}
\hline FRB & $\begin{array}{c}\text { UTC } \\
\text { detection }\end{array}$ & $\begin{array}{r}\mathrm{DM}_{\text {tot }}{ }^{a} \\
{[\mathrm{pc} /}\end{array}$ & $\begin{array}{l}\mathrm{DM}_{\mathrm{mw}}{ }^{a} \\
\left.\mathrm{~m}^{3}\right]\end{array}$ & $\begin{array}{c}t_{a r r}^{b} \\
{[\mathrm{~s}]}\end{array}$ & $\begin{array}{c}t_{\text {sweep }}{ }^{c} \\
{[\mathrm{~s}]}\end{array}$ & $\begin{array}{c}\tau_{\text {scat }}{ }^{d} \\
{[\mathrm{~ms}]}\end{array}$ & $\begin{array}{l}\mathcal{F}_{1.4 \mathrm{GHz}}^{e} \\
{[\mathrm{Jy} \mathrm{ms}]}\end{array}$ & $\begin{aligned} & \mathcal{F}_{1851} \\
\alpha= & -1\end{aligned}$ & $\begin{array}{l}{[\mathrm{Jy} \mathrm{ms}] \exp } \\
\alpha=-2\end{array}$ & $\begin{array}{l}\operatorname{ected}^{f} \\
\alpha=-1.8^{g}\end{array}$ & $\begin{array}{c}\mathcal{U}_{5 \sigma}{ }^{h} \\
{[\mathrm{Jy} \mathrm{ms}]}\end{array}$ \\
\hline 171020 & $10: 27: 59.00$ & 114.1 & 38.4 & 11.7 & 4.5 & 1.7 & $200_{-100}^{+500}$ & $1500_{-800}^{+4000}$ & $11400_{-6000}^{+30000}$ & $7600_{-4000}^{+19000}$ & 2200 \\
\hline 180110 & $07: 34: 34.95$ & 715.7 & 38.8 & 73.0 & 28.0 & 4.5 & $420_{-20}^{+20}$ & $3200_{-150}^{+150}$ & $23900_{-1100}^{+1100}$ & $16000_{-800}^{+800}$ & $\begin{array}{c}3350^{i} \\
\text { or } \\
6500^{j}\end{array}$ \\
\hline 180128.0 & $00: 59: 37.97$ & 441.4 & 31.5 & 45.0 & 17.3 & 2.9 & $51_{-2}^{+2}$ & $380_{-15}^{+15}$ & $2900_{-110}^{+110}$ & $1940_{-80}^{+80}$ & $\mathrm{GL}^{k}$ \\
\hline 180128.2 & $04: 53: 26.80$ & 495.9 & 41.0 & 50.6 & 19.40 & 2.3 & $66_{-4}^{+4}$ & $500_{-30}^{+30}$ & $3800_{-230}^{+230}$ & $2500_{-150}^{+150}$ & $\mathrm{SL}^{k}$ \\
\hline 180130 & 04:55:29.99 & 343.5 & 39.0 & 34.90 & 13.35 & 6.0 & $95_{-3}^{+3}$ & $720_{-20}^{+20}$ & $5400_{-170}^{+170}$ & $3600_{-110}^{+110}$ & $\mathrm{SL}^{k}$ \\
\hline 180315 & 05:05:30.99 & 479.0 & 101.7 & 48.66 & 18.63 & 2.4 & $56_{-4}^{+4}$ & $420_{-30}^{+30}$ & $3200_{-230}^{+230}$ & $2100_{-150}^{+150}$ & $\mathrm{SL}^{k}$ \\
\hline 180324 & 09:31:46.70 & 431.0 & 64.0 & 43.79 & 16.75 & 4.3 & $71_{-3}^{+3}$ & $540_{-20}^{+20}$ & $4000_{-170}^{+170}$ & $2700_{-110}^{+110}$ & $450^{i}$ \\
\hline
\end{tabular}

${ }^{a} \mathrm{DM}_{\text {tot }}$ : the total DM measured by ASKAP; $\mathrm{DM}_{\mathrm{mw}}$ is the contribution of the Milky Way (from NE2001; Cordes \& Lazio (2002))

${ }^{b} t_{\text {arr }}$ : the time delay between ASKAP detection at $1297 \mathrm{MHz}$, and the expected arrival at $200 \mathrm{MHz}$

${ }^{c} t_{\text {sweep }}$ : the sweep time over the MWA observing band $(170-200 \mathrm{MHz})$

$d_{\tau_{\text {scat }}}$ : scattering time at $1.4 \mathrm{GHz}$ fitted to ASKAP data for 180110 and 180130 and pulse width for other FRBs.

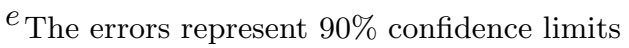

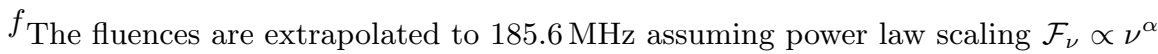

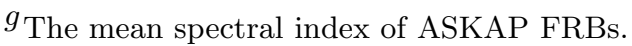

$h_{\mathcal{U}_{5 \sigma}}$ : the MWA $5 \sigma$ upper limit on $\mathcal{F}_{185 \mathrm{MHz}}$

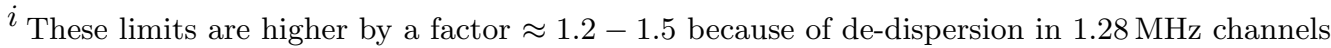

$j$ This FRB was significantly temporally broadened (see Sec. 2.3); therefore, we also present the limit from de-dispersed images averaged over $10 \mathrm{~s}$ (Tab. 2)

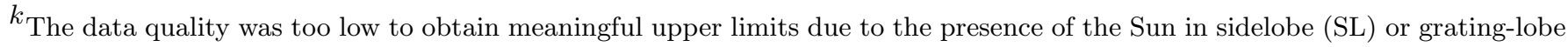
(GL)

any signs of dispersion sweep in the dynamic spectra of the candidate pixels. These time-averaged images were also visually inspected. The algorithm was executed on Stokes $\mathrm{I}$ and $\mathrm{V}$ images because noise in 0.5 -second $\mathrm{V}$ images was slightly lower $(\sim 20-30 \%)$. The above procedure resulted in upper limits on the flux densities and consequently fluences of low-frequency counterparts of three ASKAP FRBs, which are summarized in Tables 1 and 2 .

\subsection{Verification of the FRB Pipeline}

The algorithm was verified using a relatively highDM pulsar (147.29 pc cm ${ }^{-3}$ ), PSR J0837-4135, with a pulse period $\mathrm{P} \approx 0.751 \mathrm{~s}$ (Manchester et al. 2005). The pipeline detected three individual bright pulses above $5 \sigma$ in a 112 -second ( $\approx 149$ pulses) observation. Example images with and without a pulse, the dynamic spectrum and the lightcurve observed in the de-dispersed images are shown in Figure 3.

The efficiency of the algorithm was verified on a 112second observation of a lower-DM pulsar $\left(34.425 \mathrm{pc} \mathrm{cm}^{-3}\right)$, PSR J0630-2834, with $\mathrm{P} \approx 1.244 \mathrm{~s}$ (Manchester et al. 2005). In the 112-second (90 pulses) observation, the algorithm identified 31 pulses above the $5 \sigma$ threshold in the de-dispersed images (110 pulses with the $3 \sigma$ threshold). Due to lack of pulsars with a suitable combination of DM and scattering time, we have verified the algorithm on averaged images by "injecting" a simulated FRB signal into 0.5-second MWA images and confirming that it was detected in the averaged images. These tests confirmed that the algorithm is capable of detecting highly-dispersed, FRB-like transients in single and averaged 0.5-s MWA images.

\section{DISCUSSION}



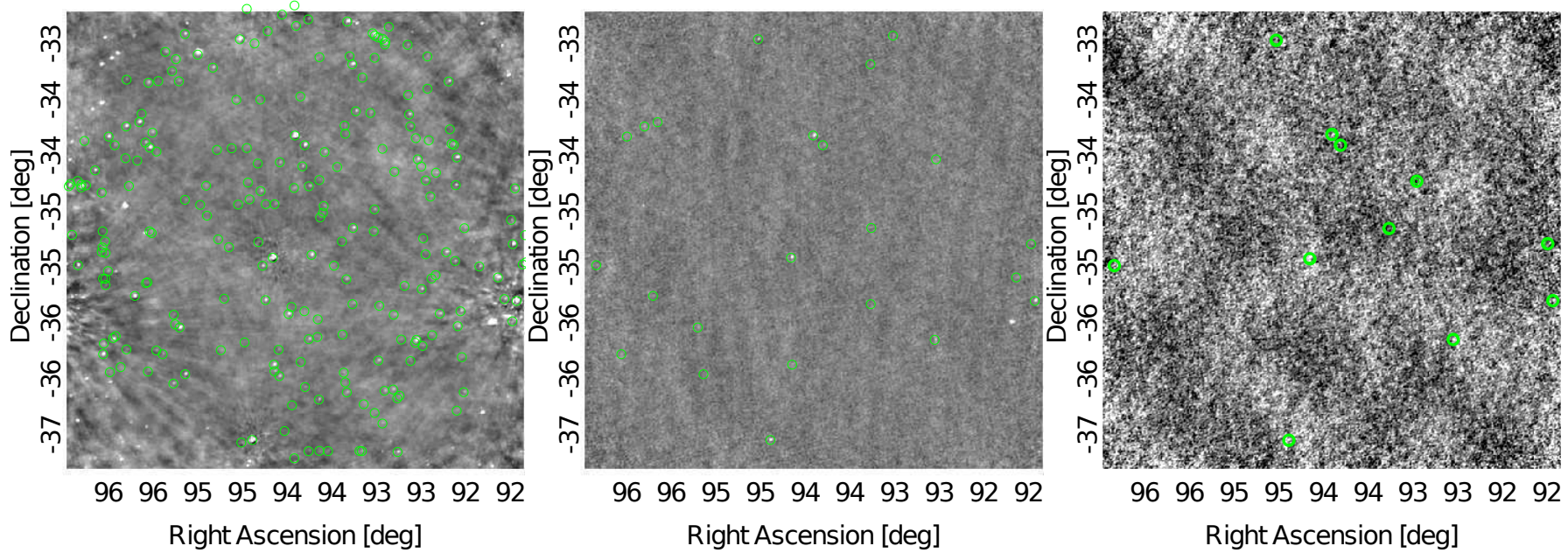

$\begin{array}{lllllllll}-2 & -1.5 & -1 & -0.5 & 0 & 0.5 & 1 & 1.5 & 2\end{array}$

Figure 2. Example images of the FRB 180324 position (image centers). (Left) The reference image of the field (the mean of 6 observations without the FRB), with noise in the center $\sigma \approx 17 \mathrm{mJy}_{\text {beam }}{ }^{-1}$; the green circles are NVSS (Condon et al. 1998) sources brighter than $10 \mathrm{mJy}$ at $1.4 \mathrm{GHz}$. The image scale spans $-0.2-0.2 \mathrm{Jy}_{\text {beam }}^{-1}$. (Center) An example 0.5 -s dirty image obtained by stacking the appropriately time-shifted $24 \times 1.28 \mathrm{MHz}$ spectral channels (i.e. de-dispersing) to $\mathrm{DM}=431.0 \mathrm{pc} \mathrm{cm}^{-3}$; the noise in the image center is $\sigma \approx 180 \mathrm{mJy}_{\text {beam }}^{-1}$ and the green circles are NVSS sources brighter than $100 \mathrm{mJy}$ at $1.4 \mathrm{GHz}$. (Right) An example single 0.5 -s dirty image in a single $1.28 \mathrm{MHz}$ channel; noise in the image center is $\sigma \approx 760 \mathrm{mJy} \mathrm{beam}^{-1}$ and the green circles are NVSS sources brighter than $200 \mathrm{mJy}$ at $1.4 \mathrm{GHz}$. The scales in the center and right images are as shown in the colorbar (in Jy beam ${ }^{-1}$ ).

The absence of low-frequency emission coincident with any of the shadowed CRAFT FRBs places constraints on the emission characteristics of these bursts and their environments. We provide a brief discussion here in the context of the four most obvious interpretations.

(i) Temporal smearing due to scattering through an inhomogeneous plasma potentially dominates over the intrinsic pulse width at low frequencies and decreases the burst detectability. An upper limit to the low-frequency pulse width is inferred by attributing the ASKAPderived pulse width entirely to scattering, and assuming that pulse broadening scales as $\nu^{-3.5}$ (or $\left.\nu^{-4}\right)^{3}$. The measured pulse widths of FRBs 171020, 180110 and 180324 at $1400 \mathrm{MHz}$ in turn imply limits on the pulse width at $185 \mathrm{MHz}$ of 2.1 (5.6), 5.4 (14.8) and 5.1 (14) seconds respectively. Since pulse fluence is unaltered by scattering, the fluence upper limits relative to the expected fluence (Tables 1 and 2) show that any pulse

3 Shannon et al. (2018) measure $\tau_{\text {scat }} \propto \nu^{-3.5 \pm 0.5}$ in the ASKAP band, so we examine the consequences of both a $\nu^{-3.5}$ and a $\nu^{-4}$ scaling. with a spectrum steeper than $\alpha \approx-1$ would have been detected. We conclude that pulse broadening alone is insufficient to explain the MWA non-detections because the spectral index of the emission would have to flatten substantially between $185 \mathrm{MHz}$ and $1295 \mathrm{MHz}$ from the mean value of $\alpha=-1.8$ measured for the ASKAP FRB population (Shannon et al. 2018).

(ii) The viability of the hypothesis that free-free absorption is responsible for the absence of low frequency emission may be cast in terms of a limit on the thickness of the absorbing material. An upper limit to the depth of the absorbing region is derived by assuming that all plasma not attributable to the Milky Way is confined to the absorbing medium (not to the intergalactic medium, thereby assuming the redshift term $(1+\mathrm{z})$ in Eq. 1 is $\approx 1)$, whose emission measure is then $\mathrm{EM}=\int N_{e}^{2} d l=\mathrm{DM}_{\text {extra }}^{2} \Delta L^{-1} \mathrm{pc} \mathrm{cm}^{-6}$, where $\Delta L$ is the region thickness in pc, $N_{e}$ is electron density, and $\mathrm{DM}_{\text {extra }}$ is the DM excess (in pc $\mathrm{cm}^{-3}$ ). A burst whose predicted flux density is $S_{\text {pred }}$ but is undetected at a limit of $S_{\lim }$ constrains the free-free optical depth to 


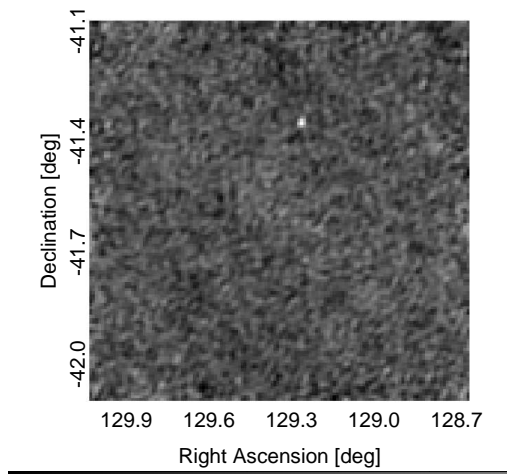

$\begin{array}{lllll}-1.5 & -1 & -0.5 & 0 & 0.5\end{array}$
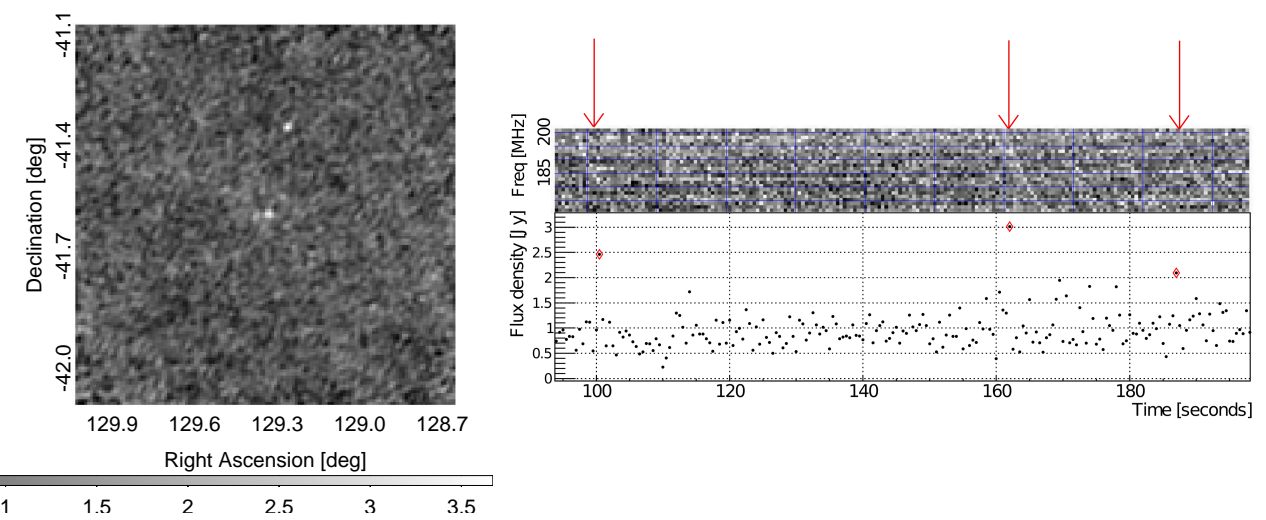

Figure 3. An example of de-dispersed images of PSR J0837-4135 (left) without a pulse, and (center) with a pulse detected by the algorithm. (Right) the corresponding dynamic spectrum of the central pixel showing the frequency sweep due to $\mathrm{DM}=147.29 \mathrm{pc} \mathrm{cm}^{-3}$ (right upper image). The three sweeps (sweep time $\approx 5.8$ seconds in the $170-200 \mathrm{MHz}$ range) can be seen at times ranges $97-105,160-166$ and $183-191$ seconds between the red arrows and the data points surrounded by the red diamonds marking the three brightest points in the lightcurve from the de-dispersed images (right lower image).

$\tau_{\mathrm{ff}}>\ln \left(S_{\text {pred }} / S_{\text {lim }}\right)$, and one has

$$
1.1 \times 10^{-5} T_{4}^{-1.35} \nu_{185}^{-2.1}\left(\frac{\mathrm{DM}_{\text {extra }}^{2}}{\Delta L}\right)>\ln \left(\frac{S_{\text {pred }}}{S_{\text {lim }}}\right)
$$

where $T_{4}$ is the electron temperature in units of $10^{4} \mathrm{~K}$ and $\nu_{185}$ is the observing frequency, normalized to $185 \mathrm{MHz}$. The redshift term is not included as the equation still leads to a valid upper limit. We assume the filling factor $f=1$, and if $f<1$ the limit is $\Delta L \propto f^{-1}$. The DM values in excess of the Milky Way contributions predicted by NE2001 (Cordes \& Lazio 2002) are 75.7, 676.9 and $367.0 \mathrm{pc} \mathrm{cm}^{-3}$ for FRBs 171020,180110 and 180324 respectively. We conservatively subtract a further $15 \mathrm{pccm}^{-3}$ for the Milky Way halo contribution (see the discussion in Shannon et al. 2018). For a burst spectral index of $\alpha=-1.8$, and using $5 \sigma$ limits for $S_{\text {lim }}$ derived from Table 2, this implies upper limits on $\Delta L$ of $3 \times 10^{-2} T_{4}^{-1.35} \mathrm{pc}, 5 T_{4}^{-1.35} \mathrm{pc}$ (using $\mathcal{U}_{5 \sigma}=6500 \mathrm{Jyms}$ ) and $0.7 T_{4}^{-1.35} \mathrm{pc}$ for these three bursts respectively. It is not possible to place significant constraint on $\tau_{\mathrm{ff}}$ for $\alpha>-1$. The tightest constraint on $\tau_{\text {ff }}$ from FRB 171020 is likely tighter because a fraction of the electron column density is associated with host galaxy interstellar medium (ISM), intergalactic medium (IGM), or both.

(iii) The flux densities of the bursts detected by ASKAP could be enhanced by plasma lensing effects due to caustics or scintillation (Cordes et al. 2017). It is improbable that any lensing effects, if present, extend over

\begin{tabular}{ccccccc}
\hline \hline $\mathrm{FRB}$ & $\sigma_{d d}$ & $\sigma_{0.5 s}$ & $\sigma_{5 s}$ & $\sigma_{10 s}$ & $\sigma_{15 s}$ & $\sigma_{20 s}$ \\
\hline 20171020 & 880 & 4570 & 400 & 350 & 330 & 310 \\
20180110 & 1340 & 6640 & 700 & 650 & 620 & 600 \\
20180324 & 180 & 840 & 120 & 120 & 110 & 110 \\
\hline \hline
\end{tabular}

Table 2. MWA $1 \sigma$ noise of flux density $\left(\mathrm{mJy}_{\mathrm{beam}}{ }^{-1}\right)$ in the centers of beam-corrected images on average of 24 dedispersed 1.28- $\mathrm{MHz}$ channels $\left(\sigma_{d d}\right)$, single 0.5 -second images $\left(\sigma_{0.5 s}\right)$ and averaged over $5\left(\sigma_{5 s}\right), 10\left(\sigma_{10 s}\right), 20\left(\sigma_{20 s}\right)$ and 30 seconds $\left(\sigma_{30 s}\right)$.

an order of magnitude in frequency, so it is unlikely that it plays a significant role both at ASKAP and low frequencies. The ratios of the measured ASKAP fluences to their $185-\mathrm{MHz}$ upper limits yield a lower limit to the magnification at $1.4 \mathrm{GHz}$. Comparing against the $5 \sigma$ upper limit at $\alpha=-1.8$, we find magnifications $\mu>3,2$ \& 6 for FRBs 171020, 180110 and 180324 respectively.

Most ASKAP FRBs exhibit fine frequency structure, some with a factor $>10$ deviations from the mean, with a filling factor of $\sim 5 \%$ (Shannon et al. 2018). If this structure is due to diffractive scintillation, the decorrelation bandwidth at $185 \mathrm{MHz}$ would be $\sim 10^{-3}$ smaller than that at $1.4 \mathrm{GHz}$ (assuming $\sim \nu^{4}$ scaling), the spectrum would therefore appear spectrally smooth at low frequency at our resolution, and the low spectral occupancy observed at $1.4 \mathrm{GHz}$ would not explain the MWA 
non-detections. However, even if the low spectral occupancy is an intrinsic feature of the emission, it is implausible to attribute the low-frequency non-detections for all three well-constrained FRBs to this effect alone, since the relative spectral bandwidths of ASKAP and the MWA are comparable at $\Delta \nu / \nu$ equal to $26 \%$ and $17 \%$ respectively.

(iv) A break in the intrinsic spectrum is a natural explanation for the non-detection and would be plausible on two grounds: (a) for a spectrum with $\alpha<-1$ the finite burst energy is dominated by the low-energy cutoff, and (b) many pulsars, whose emission resembles the properties of FRBs in some respects, also exhibit spectral breaks in the region $100-300 \mathrm{MHz}$ (see Fig. 7 of Bilous et al. (2016) or Fig. 6 of Murphy et al. (2017) for a recent summary). The limit on the spectral cutoff of $\nu_{\text {lo }}>200 \mathrm{MHz}$ for $\alpha=-1.8$ implies that the total burst energy cannot exceed 18 times more than the pulse energy measured within the ASKAP band alone.

The presented simultaneous MWA observations of extremely bright ASKAP FRB are a substantial advance over previous surveys conducted below $200 \mathrm{MHz}$. Therefore, the derived limits enable us to place tighter constraints on the physics. Indeed, no previous lowfrequency survey has a sufficiently large exposure to have detected the low-frequency counterpart to the FRBs detected by CRAFT: the $37 \pm 8$ events day $^{-1}$ sky $^{-1}$ burst detection rate at $\mathcal{F}_{1.4 \mathrm{GHz}}>26 \mathrm{Jy} \mathrm{ms}$ is equivalent to one event every $27_{-5}^{+7} \times 10^{3} \mathrm{deg}^{2} \mathrm{hr}$ (Shannon et al. 2018). This substantially exceeds the $14 \times$ $10^{3} \mathrm{deg}^{2} \mathrm{hr}$ exposure of the LOFAR Pilot Pulsar Survey at $140 \mathrm{MHz}$ (Coenen et al. 2014) with a fluence cutoff $\mathcal{F}_{\mathrm{c}}=2.75 \times 10^{3} \Delta T^{1 / 2} \mathrm{Jyms}$, for pulse widths $\Delta T \leq 1.26 \mathrm{~s}$ in the range $\mathrm{DM}=2-3000 \mathrm{pc} \mathrm{cm}^{-3}$. The ARTEMIS survey at $150 \mathrm{MHz}$ (Karastergiou et al. 2015) examined $\mathrm{DM} \leq 310 \mathrm{pc} \mathrm{cm} \mathrm{cm}^{-3}$ so would have missed 6 of the 7 FRBs reported here. Moreover, it only searched for pulse durations below $21 \mathrm{~ms}$, with a $10 \sigma$ threshold $\mathcal{F}_{\mathrm{c}}=4.47 \times 10^{3} \Delta T^{1 / 2} \mathrm{Jy} \mathrm{ms}$, where $\Delta T$ is burst width in seconds.

The blind pilot survey of Tingay et al. (2015) had an exposure of only $4.2 \times 10^{3} \mathrm{deg}^{2} \mathrm{hr}$ with a $7 \sigma$ detection limit in a de-dispersed time series of $350 \mathrm{mJy}^{\text {beam }}{ }^{-1}$ on a timescale of $2 \mathrm{~s}$ (i.e. a limiting fluence of $700 \mathrm{Jy} \mathrm{ms}$ ). The survey of Rowlinson et al. (2016) was sensitive to an event rate a factor of 8.5 lower than that of Tingay et al. (2015) but only resolved down to a time resolution of $28 \mathrm{~s}$ with a limiting fluence of $7980 \mathrm{Jy} \mathrm{ms}$.

Our results exceed limits from previous MWA observations. The $3 \sigma$ upper limit on the fluence of FRB 150418 at $185 \mathrm{MHz}$, based on shadowing of the Parkes radio telescope, is $1050 \mathrm{Jy} \mathrm{ms}$ (Keane et al. 2018). However, the Parkes burst fluence of $2.0_{-0.8}^{+1.2} \mathrm{Jy}$ ms permits only a poor constraint on the spectral index between $1382 \mathrm{MHz}$ and $185 \mathrm{MHz}$ of $\alpha>-3$.

\section{CONCLUSIONS}

We have used the MWA to attempt the co-detection of the low-frequency counterparts of the high fluence FRBs detected by the CRAFT survey. None were found. The shadowing strategy employed here enabled us to significantly reduce the searched FRB parameter volume in space, DM and time. We are able to derive strong upper limits based on the rare, very high ASKAP fluence $\left(\mathcal{F}_{1.4 \mathrm{GHz}} \gtrsim 26 \mathrm{Jy} \mathrm{ms}\right) \mathrm{FRBs}$, which would require "blind" surveys of enormous exposures $\left(\sim 27000 \operatorname{deg}^{2} \mathrm{~h}\right.$ per event) to obtain comparable limits.

No low-frequency counterparts of these FRBs were identified. The fluences of CRAFT FRBs are accurately measured, and the presented limits on broadband spectral indexes $(\alpha \gtrsim-1)$ supersede the only direct broadband limit existing in the literature $(\alpha>-3)$ obtained with the MWA and Parkes FRB 150418. These limits are strongly at variance with the mean $\alpha=-1.8 \pm 0.3$ spectral index for these FRBs measured within the ASKAP band.

We show that pulse broadening alone due to scattering is insufficient to explain the non-detections of the shadowed FRBs. We place limits on the thickness of a dense medium close to the progenitors under the assumption that free-free absorption is responsible for the spectral turnover; the tightest constraint is for FRB 171020, for which the medium thickness is $<0.03 T_{4}^{-1.35}$ pc. In this case the implied absorption region is so small that it casts considerable doubt that free-free absorption is responsible for the spectral turnover. The speed of the dense wind of any possible massive progenitor, or the shock associated with a young (older than 1 minute) SN would easily encompass a larger region than that implied here. Moreover, Mahony et al. (2018, submitted) examines the properties of the likely host galaxy of this FRB, and finds no obvious bright compact knots of continuum radio emission at frequencies up to $21 \mathrm{GHz}$ down to $31.8 \mu J y$. If FRBs are enhanced by caustics in the ASKAP band, we constrain the magnification (between 1295 and $185 \mathrm{MHz}$ ) to be $\mu>6$, in the case of FRB 180324. The largely unconstrained properties of the host galaxy ISM and intervening IGM make it difficult to assess the viability of these implied lensing magnifications. However, the analysis in Macquart et al. (2018, submitted) shows that the spectral structure of the bursts at $1.4 \mathrm{GHz}$ appears to be qualitatively inconsistent with the effect of plasma lensing, which disfavors lensing as the explanation of the MWA non-detections. 
A turn-over in the intrinsic FRB spectrum, however, remains a plausible explanation.

For $\alpha \leq-1$ the low-frequency cutoff dominates the burst energy. Thus, for $\alpha=-1.8$ our non-detections limit the total burst energy to $<18$ times the $\sim 10^{35} \mathrm{~J}$ values deduced for FRB detections in the ASKAP and Parkes bands alone.

To date, no FRB has been detected simultaneously at high and low frequencies. The on-going enhancements to the MWA will improve the detection sensitivity for FRB searches by about an order of magnitude (owing to much higher temporal and spectral resolutions possible via the voltage buffer mode). These improvements will significantly increase the prospects of detecting low-frequency emission if it is much fainter than our currently set limits, which may warrant a review of FRB models and physics.

This scientific work makes use of the Murchison Radio-astronomy Observatory (MRO), operated by CSIRO. We acknowledge the Wajarri Yamatji people as the traditional owners of the Observatory site. Support for the operation of the MWA is provided by the
Australian Government (NCRIS), under a contract to Curtin University administered by Astronomy Australia Limited. The ASKAP is part of the Australia Telescope National Facility which is managed by CSIRO. Operation of ASKAP is funded by the Australian Government with support from the National Collaborative Research Infrastructure Strategy. ASKAP uses the resources of the Pawsey Supercomputing Centre. Establishment of ASKAP, the MRO and the Pawsey Supercomputing Centre are initiatives of the Australian Government, with support from the Government of Western Australia and the Science and Industry Endowment Fund. JPM, RMS \& KWB acknowledge Australian Research Council grant DP180100857. R.M.S. acknowledges salary support through Australian Research Council (ARC) grants FL150100148 and CE170100004. Parts of this research were supported by the Australian Research Council Centre of Excellence for All Sky Astrophysics in 3 Dimensions (ASTRO 3D), through project number CE170100013. Parts of this research were conducted by the Australian Research Council Centre of Excellence for All-sky Astrophysics (CAASTRO), through project number CE110001020.

\section{REFERENCES}

Amiri, M., Bandura, K., Berger, P., et al. 2017, ApJ, 844, 161

Bannister, K. W., Shannon, R. M., Macquart, J.-P., et al. 2017, ApJL, 841, L12

Bhat, N. D. R., Cordes, J. M., Camilo, F., Nice, D. J., \& Lorimer, D. R. 2004, ApJ, 605, 759

Bilous, A. V., Kondratiev, V. I., Kramer, M., et al. 2016, A\&A, 591, A134

Boyle et al., P. 2018, The Astronomer's Telegram, 11901

Burke-Spolaor, S., Trott, C. M., Brisken, W. F., et al. 2016, ApJ, 826, 223

Chawla, P., Kaspi, V. M., Josephy, A., et al. 2017, ApJ, 844,140

Coenen, T., van Leeuwen, J., Hessels, J. W. T., et al. 2014, A\&A, 570, A60

Condon, J. J., Cotton, W. D., Greisen, E. W., et al. 1998, AJ, 115, 1693

Cordes, J. M., \& Lazio, T. J. W. 2002, ArXiv Astrophysics e-prints, astro-ph/0207156

Cordes, J. M., Wasserman, I., Hessels, J. W. T., et al. 2017, ApJ, 842, 35

Fender, R., Stewart, A., Macquart, J. P., et al. 2015a, Advancing Astrophysics with the Square Kilometre Array (AASKA14), 51
Fender, R., Stewart, A., Macquart, J. P., et al. 2015b, in Advancing Astrophysics with the Square Kilometre Array (AASKA14) (Proceedings of Science), 51

Gajjar, V., Siemion, A. P. V., Price, D. C., et al. 2018, ArXiv e-prints, arXiv:1804.04101

Haslam, C. G. T., Salter, C. J., Stoffel, H., \& Wilson, W. E. 1982, A\&AS, 47, 1

Hurley-Walker, N., Callingham, J. R., Hancock, P. J., et al. 2017, MNRAS, 464, 1146

Karastergiou, A., Chennamangalam, J., Armour, W., et al. 2015, MNRAS, 452, 1254

Keane, E. F., Johnston, S., Bhandari, S., et al. 2016, Nature, 530, 453

Keane, E. F., Barr, E. D., Jameson, A., et al. 2018, MNRAS, 473, 116

Law, C. J., Abruzzo, M. W., Bassa, C. G., et al. 2017, ApJ, 850,76

Lorimer, D. R., Bailes, M., McLaughlin, M. A., Narkevic, D. J., \& Crawford, F. 2007, Science, 318, 777

Macquart, J.-P., Bailes, M., Bhat, N. D. R., et al. 2010, PASA, 27, 272

Macquart et al., J.-P. 2018, submitted

Mahony et al., E. 2018, submitted

Manchester, R. N., Hobbs, G. B., Teoh, A., \& Hobbs, M. 2005, AJ, 129, 1993 
Masui, K., Lin, H.-H., Sievers, J., et al. 2015, Nature, 528, 523

McMullin, J. P., Waters, B., Schiebel, D., Young, W., \& Golap, K. 2007, in Astronomical Society of the Pacific Conference Series, Vol. 376, Astronomical Data Analysis Software and Systems XVI, ed. R. A. Shaw, F. Hill, \& D. J. Bell, 127

Murphy, T., Kaplan, D. L., Bell, M. E., et al. 2017, PASA, 34, e020

Offringa, A. R., McKinley, B., Hurley-Walker, N., et al. 2014, MNRAS, 444, 606

Petroff, E., Keane, E. F., Barr, E. D., et al. 2015, MNRAS, 451, 3933

Rowlinson, A., Bell, M. E., Murphy, T., et al. 2016, MNRAS, 458, 3506

Scholz, P., Spitler, L. G., Hessels, J. W. T., et al. 2016, ApJ, 833, 177
Shannon, R., Macquart, J.-P., Bannister, K., \& Ekers, R. D. 2018, Nature, 474, arXiv:1804.???, accepted

Sokolowski, M., Colegate, T., Sutinjo, A. T., et al. 2017, PASA, 34, e062

Spitler, L. G., Scholz, P., Hessels, J. W. T., et al. 2016, Nature, 531, 202

The CHIME/FRB Collaboration, :, Amiri, M., et al. 2018, ArXiv e-prints, arXiv:1803.11235

Thornton, D., Stappers, B., Bailes, M., et al. 2013, Science, 341,53

Tingay, S. J., Goeke, R., Bowman, J. D., et al. 2013, PASA, 30, e007

Tingay, S. J., Trott, C. M., Wayth, R. B., et al. 2015, AJ, 150, 199

Wayth, R. B., Tingay, S. J., Trott, C. M., et al. 2018, ArXiv e-prints, arXiv:1809.06466 